\title{
Spatiotemporal evolution of light during propagation in filamentation regime
}

\author{
Benjamín Alonso, ${ }^{1}$ Íñigo J. Sola, ${ }^{1, *}$ Julio San Román, ${ }^{1}$ Óscar Varela, ${ }^{2}$ and Luis Roso ${ }^{2}$ \\ ${ }^{1}$ Universidad de Salamanca, Grupo de Investigación en Óptica Extrema (GIOE), \\ Plaza de la Merced, s/n E-37008 Salamanca, Spain \\ ${ }^{2}$ Centro de Láseres Pulsados (CLPU), E-37008 Salamanca, Spain \\ ${ }^{*}$ Corresponding author: ijsola@usal.es
}

Received January 19, 2011; revised May 14, 2011; accepted May 26, 2011; posted June 6, 2011 (Doc. ID 141272); published July 5, 2011

\begin{abstract}
The full description of the evolution of light during its nonlinear propagation represents a valuable help to the complete understanding of important nonlinear phenomena such as light filamentation. In this paper we present a comparison between theoretical and experimental results of the spatiotemporal structure of a light filament at different propagation distances. In order to obtain the experimental spatiotemporal structure, we have used a technique based on spatially resolved spectral interferometry called STARFISH, for spatiotemporal amplitudeand-phase reconstruction by Fourier transform of interference spectra of high-complex beams. We have been able to observe important nonlinear pulse dynamics during the nonlinear propagation, including pulse splitting and the subsequent competition among the pulses that result from the splitting, obtaining a full insight into the general nonlinear behavior. (C) 2011 Optical Society of America OCIS codes: $\quad 320.7110,320.7100$.
\end{abstract}

\section{INTRODUCTION}

The advent of high-power pulsed lasers in the last decades has allowed the study of the propagation of light in nonlinear regime. Several different behaviors have been observed; among others, the modified interference pattern of light in the two-slit experiment [1] and the self-guiding propagation of light when diffraction and the Kerr effect compete, with no ionization of the medium [2-4]. But the process that has attracted more attention in the past years is the so-called filamentation of light. This is a regime of nonlinear propagation where the equilibrium between the self-focusing process, caused by the Kerr effect, and the self-defocusing process, induced by the presence of weak plasma generated by the pulse, leads to the self-guiding of the light [5-7]. This phenomenon has been applied to pulse postcompression [8], atmospheric analysis [9,10], remote LIBS [11], and the generation of subterahertz radiation [12], for a brief list of examples.

The filamentation regime has been studied extensively by means of theoretical simulations considering different media, showing the high degree of complexity of the behavior of the electric field in the spatiotemporal domain $[6,13,14]$. The experimental observation of these dynamics and the total understanding of the phenomena remain open problems [15]. An important amount of work and effort has been devoted to further experimental comprehension of the different nonlinear phenomena. Several methods have been used to obtain further information about the filamentation process, such as measurement of the electric conductivity of the medium [16] or spectroscopy of the plasma emission for analyzing the electronic density and the temperature of the plasma [17], measurements of the high orders of the nonlinear refraction index [18], and reconstruction of the pulses at different points of the transverse plane to obtain the local temporal structure [19].
To date, the experimental evidence and studies of pulse dynamics have come from techniques that partially describe the beam propagation. Minardi et al. used the shadowgraphy technique, obtaining the refractive index and the absorption of the medium when the probe pulse passed through the filament [20]. From these results it was possible to observe some features of the beam propagation dynamics, such as the separation velocity of the two split pulses. Another observation of the spatiotemporal dynamics, obtaining experimental evidence of the pulse splitting, was obtained by using femtosecond time-resolved optical polarigraphy [21]. This technique uses a probe beam to detect the birefringence generated by a filament acting as a pump. In addition, some traces of asymmetrical pulse splitting could be observed.

On the other hand, the spatiotemporal reconstruction of light pulses has been notably developed in the past few years by the introduction of several techniques [22-30]. Therefore, the tools for the experimental study of the spatiotemporal structure of the beam propagating nonlinearly are already available. The first important approach for the spatiotemporal reconstruction of nonlinearly propagated beams was performed by using spatially resolved cross correlation [22-24]. It consisted of spatially resolved temporal cross correlation of the spatiotemporal intensity. The test pulse was temporally and spatially probed for different delays by a shorter known pulse. The information given by this technique is very valuable, showing general features of the nonlinear generation of $X$-type waves during the propagation of a femtosecond pulse along a nonlinear crystal [22] or the pulse splitting dynamics when propagating a femtosecond pulse through water [23]. However, the spectral phase remained unknown, so the information was still partial. The aim of the present work is to achieve a full spatiotemporal characterization of the electric field of a filament at different planes along its propagation. This will give a more complete understanding of the splitting 
and self-compression dynamics, since it will describe the evolution of the pulse fronts, the spectral spatiotemporal distribution, and the time-space coupling effects. For this purpose, we used spatiotemporal amplitude-and-phase reconstruction by Fourier transform of interference spectra of high-complex beams (STARFISH [30]), a simple reconstruction technique already tested for the complete characterization of spatiotemporally complex pulses in the linear regime [31]. The experimental results have been contrasted with the established theory that describes filament formation.

During the preparation of this manuscript, we have received knowledge of an attempt at filament reconstruction [32] also based on a spectral interferometry reconstruction setup.

This paper is structured as follows: in Section 2 we present the tools employed during this work on the experimental side; we comment on the STARFISH technique and its implementation for the experimental measurements. In Section 3, the theoretical model used for the present work is described. Section $\underline{4}$ is devoted to the presentation and analysis of the experimental results and their comparison with the theoretical simulations. Finally, the conclusion summarizes the most important ideas obtained from the work.

\section{EXPERIMENTAL SETUP}

All the experimental results presented here were obtained with a Spectra-Physics chirped pulse amplification (CPA) Ti:sapphire laser (Newport Corporation, USA), delivering $100 \mathrm{fs}$ pulses (central wavelength at $795 \mathrm{~nm}, 10 \mathrm{~nm}$ FWHM), $10 \mathrm{~Hz}$ repetition rate, and $9 \mathrm{~mm}$ diameter beam. In order to study the filamentation process in air, we reconstructed the spatiotemporal structure of the beam at different propagation distances using the STARFISH characterization method [30], based on spectral interferometry [33] of an unknown test pulse and a reference pulse [34]. In STARFISH, an optical fiber coupler is used as an interferometer: one fiber input takes the reference pulse \{previously characterized by standard reconstruction techniques, such as spectral phase interferometry for direct electric-field reconstruction (SPIDER) [35] or frequency-resolved optical gating (FROG) [36] \}, while the second one takes different spatial samples of the unknown pulse, scanning it spatially. Then, both pulses are combined in the optical fiber coupler that is directly connected to a standard spectrometer. The two pulses are delayed in time in order to obtain spectral interferences encoding the spectral phase difference between the two pulses. By employing fringe inversion techniques, such as Fourier transform spectral interferometry, proposed by Lepetit et al. [34], it is possible to reconstruct the test pulse at each scanned spatial position (whenever the reference pulse phase is characterized). Since the test pulse phases at the different spatial scan positions are referred to the same reference pulse, the full spatiotemporal information is obtained in the pulse reconstruction.

The experimental implementation for the spatiotemporal characterization of the filament is shown in Fig. 1. The pulse coming from a CPA laser system was divided with a beam splitter to generate the reference pulse and the filament (the test pulse). To create an adequate reference pulse, the beam transmitted through the beam splitter passed through a $1.5 \mathrm{~m}$ focal length lens, and it was coupled into a hollow core fiber (300 $\mu \mathrm{m}$ inner diameter, $40 \mathrm{~cm}$ long) filled with air at at- mospheric pressure. The beam was guided into the hollow core fiber, where it experienced nonlinear spectral broadening due to self-phase modulation (SPM). An iris placed just before the focusing lens was used to optimize the beam coupling into the hollow core fiber. The spectral broadening can be controlled by modifying the input pulse energy and chirp. This is especially useful when adjusting the reference spectrum to the test pulse spectrum, since, as is well known, reconstruction by spectral interferometry needs to have the reference spectrum covering the test; otherwise, the information concerning the test spectral phase in the noninterfering regions will be lost. Therefore, we focused on having a stable reference pulse whose spectrum fully covered the spectrum corresponding to that of the test pulse instead of obtaining the shortest pulse possible with this setup. Then the reference beam coming out of the hollow fiber was collimated by a $40 \mathrm{~cm}$ lens and passed through a transmission pair-of-grating pulse compressor to compensate the residual phase of the pulses. By means of a flip mirror, the beam could be driven into a second harmonic generation (SHG) FROG device (GRENOUILLE [37] from Swamp Optics, USA), to obtain the temporal reconstruction of the pulse, achieving typically around 24 fs pulse durations (FWHM). The spectral phase compensation was used mainly to achieve pulse durations within the time range of the GRENOUILLE. The ambiguity concerning the time direction in the SHG FROG was solved by means of additional measurements just introducing windows with known dispersion. Once the pulse was known, the mirror mentioned before was flipped and the pulse was directed into one of the two inputs of a fiber coupler. This input was located at a fixed position, providing a constant reference.

The second arm of the interferometer, which comes from the light reflected by the beam splitter, was used to create the experiment under study (the test pulse). In our case, the beam was also focused by a $1.5 \mathrm{~m}$ focal length lens to generate a filament in air. An iris just before the focusing lens was used to control the filamentation process (e.g., obtaining a good central mode). After the iris, fixed to $4 \mathrm{~mm}$ diameter during the whole experiment, the energy of the beam was $0.7 \mathrm{~mJ}$. The analysis of the beam at a given propagation distance from the lens, $z$, was performed by sending into the STARFISH the reflection of a small fraction of the energy of the beam on a plate at that position. We have checked that, although the part of the pulse transmitted inside the plate damaged the bulk, the plate surface remained undamaged (preserving its reflection and flatness) even for the higher intensities used in these experiments. The beam sampled at the plate was imaged by a $4 f$ optical system $(f=20 \mathrm{~cm})$ into the fiber of the STARFISH devoted to taking the test signal. The optical fiber coupler recombined the test spectrum with the reference spectrum and, after selecting a correct delay between both pulses (just moving the reference optical fiber back or forward), it was possible to obtain the interference in the total spectrum (test and reference). By simply moving the second optical fiber within the image plane (this does the spatial transverse scan), it was then possible to obtain the complete spatiotemporal reconstruction of the test beam. If the physical system under study presents cylindrical symmetry (which is the case of the measurements presented in this work), a radial scan is sufficient.

In order to study the filament propagation dynamics, the lens and the iris of the test arm were moved along the 


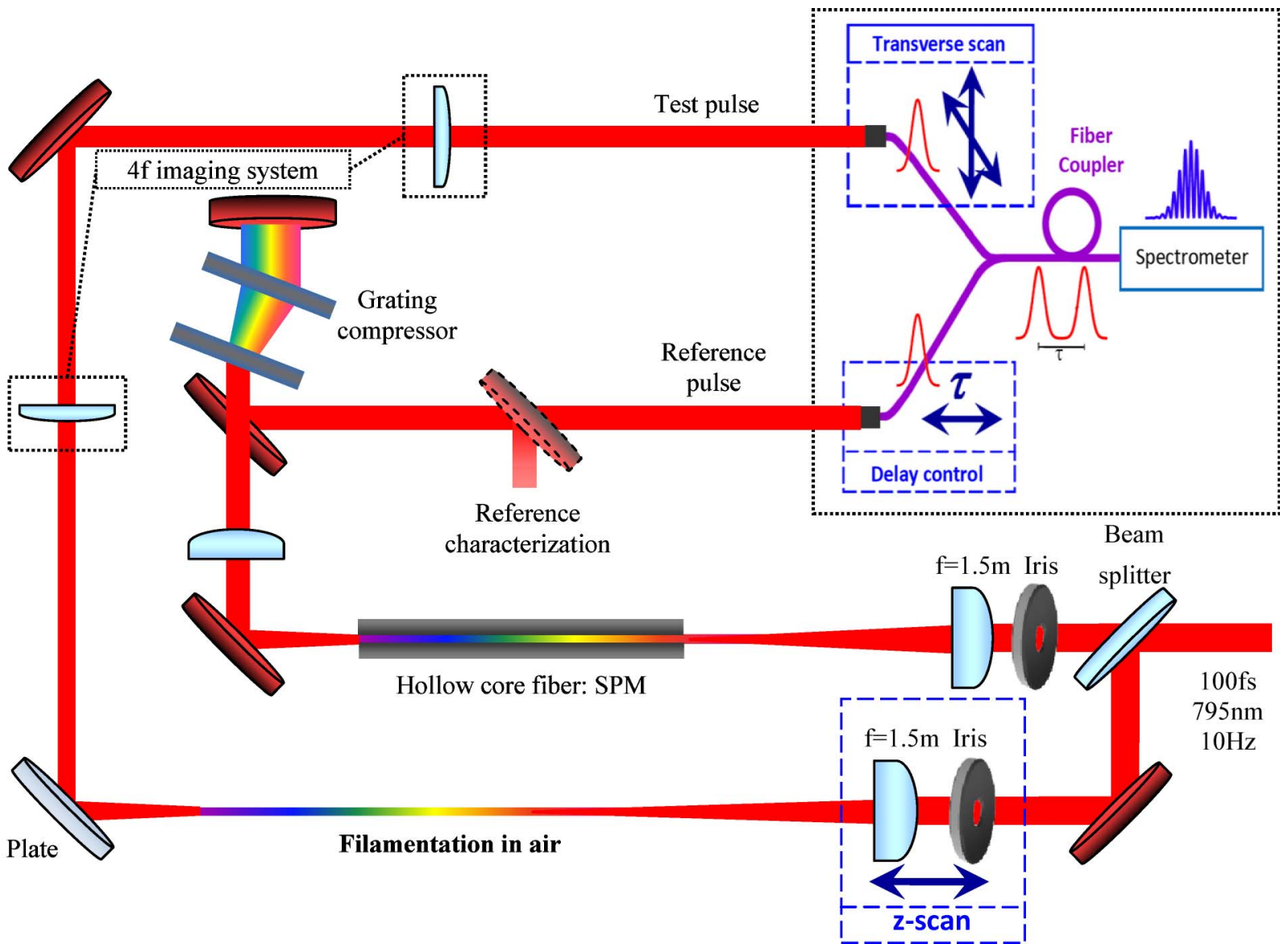

Fig. 1. (Color online) Experimental setup used for the spatiotemporal study of the filamentation process. The input laser is divided into two beams. The first pulse spectrum is broadened into a hollow core fiber. Then it is compressed and characterized. The second pulse produces filamentation in air, which is sampled by a reflective plate and imaged into the detection. The filament is characterized by the STARFISH technique: an optical fiber coupler collects both pulses to perform spectral interferometry. The propagation of the filament is tracked in a $z$-scan by moving the lens and iris along the optical axis. SPM, self-phase modulation.

propagation direction, thus changing the position under analysis while maintaining the relative delay between both arms. The experiment configuration gives a high degree of flexibility, robustness, and simplicity to the detection setup.

Since the measurement process is multishot, due to both the propagation and the transverse scans, the stability of the physical system and the reconstruction setup is a key point. Therefore, we assured that the reference and test beam were stable by checking that the reference pulse and the spatiotemporal reconstruction of the filament did not vary shot to shot. Concerning the test beam, we averaged 20 shots of the spectral amplitude in order to reduce the noise of the reconstructions. For the same purpose, we recorded 20 shots of spectral interferences at each spatial transverse position and averaged the phase retrieval, verifying that the spatiotemporal structure of the filament was maintained shot to shot and when doing the average.

\section{THEORETICAL MODEL}

The theoretical model used here to understand the spatiotemporal dynamics appearing during the nonlinear propagation is the extended nonlinear propagation equation for the envelope of the pulse $(\varepsilon)[\underline{38}, \underline{39}]$ :

$$
\begin{aligned}
\frac{\partial \varepsilon}{\partial z} & =\frac{i}{2 k_{0}}\left(1+\frac{i}{\omega_{0}} \frac{\partial}{\partial t}\right)^{-1}\left(\frac{\partial^{2}}{\partial r^{2}}+\frac{1}{r} \frac{\partial}{\partial r}\right) \varepsilon \\
& +\sum_{n=2}^{\infty} \frac{i^{n+1}}{n !}\left(\frac{\partial^{n} k}{\partial \omega^{n}}\right)_{\omega_{0}} \frac{\partial^{n} \varepsilon}{\partial t^{n}}+T_{\mathrm{NL}}(\varepsilon),
\end{aligned}
$$

where $k_{0}$ is the wave number associated with the central frequency of the pulse $\omega_{0}, z$ is the propagation distance, $r$ is the radial coordinate, and $t$ is the time. The first term of the righthand side of Eq. (1) represents the diffraction, including also the space-time coupling term with the presence of the temporal derivative. The second term represents the dispersion, while the third term includes the principal nonlinear terms that affect the propagation of the pulse, $T_{\mathrm{NL}}(\varepsilon)=K(\varepsilon)+$ $I(\varepsilon)+A(\varepsilon)$, where $K(\varepsilon)$ corresponds to the Kerr effect, $I(\varepsilon)$ corresponds to the ionization of the medium, and $A(\varepsilon)$ corresponds to the nonlinear absorption. The expressions used for these nonlinear terms are the following:

$$
\begin{aligned}
K(\varepsilon)= & i k_{0} n_{2}\left(1+\frac{i}{\omega_{0}} \frac{\partial}{\partial t}\right)\left[\left(\left(1-x_{D K}\right)|\varepsilon|^{2}\right.\right. \\
& \left.\left.+\frac{x_{D K}}{\tau_{D K}} \int_{-\infty}^{t} e^{\frac{\tau-t}{\tau_{D K}}}|\varepsilon(\tau)|^{2} \mathrm{~d} \tau\right) \varepsilon\right],
\end{aligned}
$$




$$
\begin{gathered}
I(\varepsilon)=-i \frac{k_{0}}{2 \rho_{C}}\left(1+\frac{i}{\omega_{0}} \frac{\partial}{\partial t}\right)^{-1}[\rho \varepsilon], \\
A(\varepsilon)=-\frac{1}{2} \frac{\sum_{n} \rho_{a t} W_{n} K_{n} \hbar \omega_{0}}{|\varepsilon|^{2}} \varepsilon .
\end{gathered}
$$

As shown in Eq. (2), the Kerr term includes both the instantaneous and the retarded contribution related to the Raman scattering of the molecules present in the air, where $n_{2}$ is the nonlinear index of refraction, $x_{D K}$ is the weight of the Raman contribution, and $\tau_{D K}$ is the characteristic time related to the Raman response. Moreover, the model includes the selfsteepening effect, which may be important in situations where short structures are generated during propagation. Similarly to the self-steepening, the model includes a temporal effect on the ionization. In the second term, described in Eq. (3), $\rho_{C}$ represents the critical electron density of the medium. Of course, it is necessary to compute the evolution of the ionization to complete the description of the propagation of the pulse along the medium with the following equation:

$$
\frac{\partial \rho}{\partial t}=\sum_{n} W_{n} \rho_{n}
$$

where $\rho$ and $\rho_{n}$ represent the electron density and the density of the neutral component of the gas (oxygen and nitrogen in our case), respectively. $W_{n}=\sigma_{n} I^{K_{n}}$, where $\sigma_{n}$ and $K_{n}$ are the ionization rates and the minimum number of photons needed to ionize the different components of the medium, respectively. Finally, the absorption term, described in Eq. (4), takes into account the energy that the pulse dedicates to ionize the medium, where $\rho_{a t}$ is the initial density of neutral components. For the ionization calculation, we assumed that the multiphoton ionization (MPI) is the dominant process, as supported by previous works [ $\underline{40}-43]$; thus we have not taken into account either the avalanche ionization or the electron recombination. The avalanche ionization can be discarded for subpicosecond pulses [44], and the electron recombination is also a very small correction to the ionization dynamics for our parameters. Both contributions were numerically demonstrated to be negligible.

The envelope that we have used in the model as the input pulse is a supergaussian radial profile with an experimental temporal profile, $f(t)$, that we obtained from experimental characterization of the input conditions:

$$
\varepsilon(r, t, z=0)=E_{0} \exp \left[-\left(\frac{r}{w_{0}}\right)^{4}\right] \exp \left[-i \frac{k r^{2}}{2 f^{\prime}}\right] f(t) .
$$

The supergaussian spatial profile was used in order to be close to the experimental observation of the spatial distribution of the beam. The moderate spectral bandwidth and the soft focusing induced by the aperture allow the use of the focusing phase factor as shown in Eq. (6), instead of a more accurate description as in [45], with no appearance of artificial effects.

The parameters that we have used for the simulations are the following: the beam input spatial radius, $w_{0}$, is $2 \mathrm{~mm}$; the pulse is focused with a $1.5 \mathrm{~m}$ focal length lens; its spectrum is centered at $795 \mathrm{~nm}$; and it has an input energy of $0.7 \mathrm{~mJ}$. The temporal envelope used in the simulations, obtained from the experiments, is shown in Fig. 2. The medium where the pulse propagates is air, and to describe it we have used the dependence of the refractive index, $n(\lambda)$, calculated in [46]. The value for the nonlinear refractive index is $n_{2}=$ $3.2 \cdot 10^{-19} \mathrm{~cm}^{2} / \mathrm{W}$, being equal to the contribution from the electronic part rather than the contribution from the Raman part $\left(x_{D K}=0.5\right)$ and $\tau_{D K}=70 \mathrm{fs}$. To evaluate the ionization of the air, we used the following parameters: the number of photons required to ionize the oxygen and nitrogen are $K_{\mathrm{O}_{2}}=$ 8 and $K_{N_{2}}=10$, respectively; and the corresponding ionization cross sections are $\sigma_{O_{2}}=3.5107 \cdot 10^{-96} \mathrm{~s}^{-1} \mathrm{~cm}^{16} / \mathrm{W}^{8}$ and $\sigma_{N_{2}}=9.4829 \cdot 10^{-127} \mathrm{~s}^{-1} \mathrm{~cm}^{20} / \mathrm{W}^{10}$. The MPI coefficients were calculated by using the Perelomov-Popov-Terent'ev model as presented in [6]. Finally, the critical density and the initial density of neutral molecules are $\rho_{C}=1.8 \cdot 10^{21} \mathrm{~cm}^{-3}$ and $\rho_{a t}=2.7 \cdot 10^{19} \mathrm{~cm}^{-3}$, respectively.
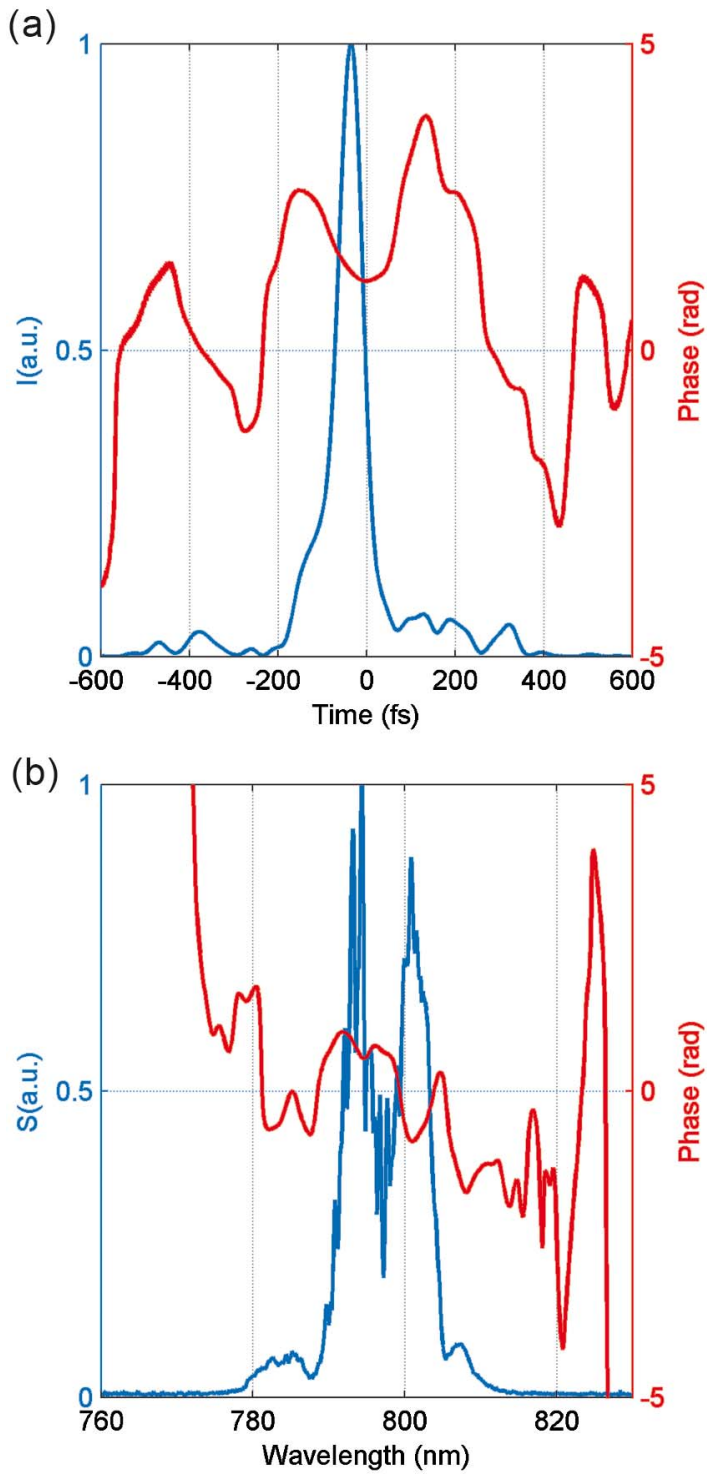

Fig. 2. (Color online) (a) Temporal and (b) spectral structure of the input pulse used in the simulations. On both plots, the blue curve represents the intensity of the envelope, while the red one corresponds to the phase. This particular temporal shape comes from the fact that the pulse shows a slight nonlinear propagation while going from the compressor to the experimentation table. 


\section{EXPERIMENTAL RESULTS AND COMPARISON WITH SIMULATIONS}

The spatiotemporal reconstruction of the beam at different propagation distances is presented in Fig. 3, where we show different snapshots of the beam during its propagation after the lens. In the first column, labeled "A," we present the experimental pulse spectra at different transverse positions $(x)$ and at a given propagation position from the focusing lens $(z)$, indicated on the left side of Fig. 3. In the second column, labeled "B," we show the corresponding spatio-spectral distributions obtained from simulations. The experimental spatiotemporal reconstructions of the pulse front at the corresponding propagation position are presented in the third column ("C"), while the theoretical spatiotemporal distributions are shown in the fourth column ("D"). For the sake of clearness, we refer to each subplot by its column label (from A to D) and its $z$ coordinate (e.g., the experimental spatiotemporal reconstruction contained in column $\mathrm{C}$ at $z=160 \mathrm{~cm}$ after the focusing lens will be denoted "C-160 cm"). We have plotted the figures in logarithmic scale to appreciate the different structures (e.g., core part, wings, pre- and postpulses, etc.) properly.

In order to interpret the results and to compare them with previous works, developed mainly on-axis, we have plotted in Fig. $\underline{4}$ the on-axis pulse reconstruction $(E(t, x=0))$ for each of the analyzed propagation positions $z$ both for the experiments (column A) and for the theory (column B). The instantaneous frequency, obtained from the first derivative of the field phase on the time domain, is shown in the colored filling of the intensity profile. Again, we refer to each subplot by the column label and the $z$ position.

First, before the nominal focus, at $z=120 \mathrm{~cm}$ after the lens, the beam starts to present some signals related to the nonlinear propagation that has happened until that position. The spectrum shows some broadening in the central part of the beam (Fig. 3, subplots A-120 cm and B-120 cm) and the pulse front basically shows some structure of a train of pre- and postpulses, which mainly comes from the input temporal structure (Fig. 3, subplots C-120 cm and D-120 cm). In its on-axis reconstruction (Fig. 4), the input pulse main structures remain without relevant changes, both for the case of the simulation and also for the experimental data (see Fig. $\underline{4}$, subplots A-120 cm and B-120 cm).

Some differences between the experimental and the theoretical data can already be observed at $z=120 \mathrm{~cm}$. The main difference is the spatial size of the pulse, which is larger in the experiment. Several reasons could be the origin of this effect. One may be the presence of some wavefront aberrations in the experimental case, especially a slight astigmatism observed when analyzing the beam with a wavefront measurement device from Phasics, France. The presence of the astigmatism, which was not included in the simulations to avoid breaking the cylindrical symmetry, is consistent with the fact that the beam size evolution within the studied propagation region is slightly smoother in the experimental results than in the simulations. This is understood by the softer linear focusing of a propagating astigmatic beam and the related increase of critical power for self-focusing in a nonlinear regime that is our case [47]. In addition, the input beam presents $M^{2}=1.2$, so the linear focus would be bigger than the focus corresponding to the ideal case. As a conse- quence of the more intense collapse achieved in the simulations, the spectral broadening and also the divergence obtained at larger distances are larger than in the experiments.

Just before the nominal focus, at $z=140 \mathrm{~cm}$, the beam begins to show the main changes. The spectrum presents the largest broadening, as is shown in Fig. 3, subplots A-140 cm and $\mathrm{B}-140 \mathrm{~cm}$. Together with this spectral broadening, an incipient temporal pulse splitting appears in the central part of the beam, while some structures become visible in the outer part of the beam (see Fig. 3, subplots C-140 cm and D-140 cm). At this stage, a self-compression process is observed. When the beam has passed the nominal focus, at $z=160 \mathrm{~cm}$, we can observe that the maximum spectral broadening is almost achieved, and minor changes can be observed in the spectra. In contrast, the spatiotemporal structure is gaining a lot of complexity, showing a clear temporal pulse splitting at the center of the beam, while the spatial wings of the pulse exhibit temporal modulations (see Fig. $\underline{3}$, subplots C-160 cm and D-160 cm).

At distances further than $z=160 \mathrm{~cm}$, the beam starts to diverge, showing an increase of its size while increasing the propagation distance. Meanwhile, the spectra, which present a quite modulated structure, show some changes of the structure of the different maxima that are present in the central spatial part. Leaving aside these details, the experimental and theoretical data show the general structure of the spectra (very modulated) that can be explained by taking into account that the Kerr effects (the instantaneous and the retarded) are active during a much longer period of the propagation than the ionization, thus dominating the process.

Figure $\underline{5}$ shows the theoretical electronic density generated by the pulse. As can be observed, ionization occurs only in a small region of about $30 \mathrm{~cm}$ around the nominal focus. Out of this region, the Kerr effect enhances the well-contrasted maxima in the spectrum, whereas the presence of the retarded contribution induces a redshift, which is basically the observed dynamics. It is interesting to note that according to Fig. 5, the main electronic densities (i.e., the plasma region) appear between $z=125 \mathrm{~cm}$ and $z=150 \mathrm{~cm}$, precisely before the region where the pulse splitting is observed, both theoretically and experimentally, which is consistent with the role of the plasma in the pulse splitting: the trail part of the pulse would suffer from plasma defocusing, triggering an asymmetric behavior on the time domain and inducing an intrinsic split of the pulse $[\underline{40}, \underline{48}, \underline{49}]$.

As mentioned previously, some of the temporal dynamics of the propagation are shown more clearly in the on-axis reconstruction plots shown in Fig. 4. The general evolution in both cases, theoretical and experimental, is similar, presenting a temporal pulse splitting and its subsequent evolution. Once the pulse splitting occurs, there is a competitive dynamics between the two main split pulses. When the beam propagates further (from $z=180 \mathrm{~cm}$ in the simulations, from $\boldsymbol{z}=220$ in the experiments) one of the split pulses, apparently the first one (the reddish), fades out until becoming a pedestal before the main pulse. It is worth comparing this result with some works already published, such as Ref. [50], although the experimental conditions are different (filamentation in argon- an atomic medium - of $40 \mathrm{fs}$ pulses, while in our case, it is filamentation on air - a molecular medium-of longer pulses, $100 \mathrm{fs}$ ). In that work, the authors presented theoretical 
A
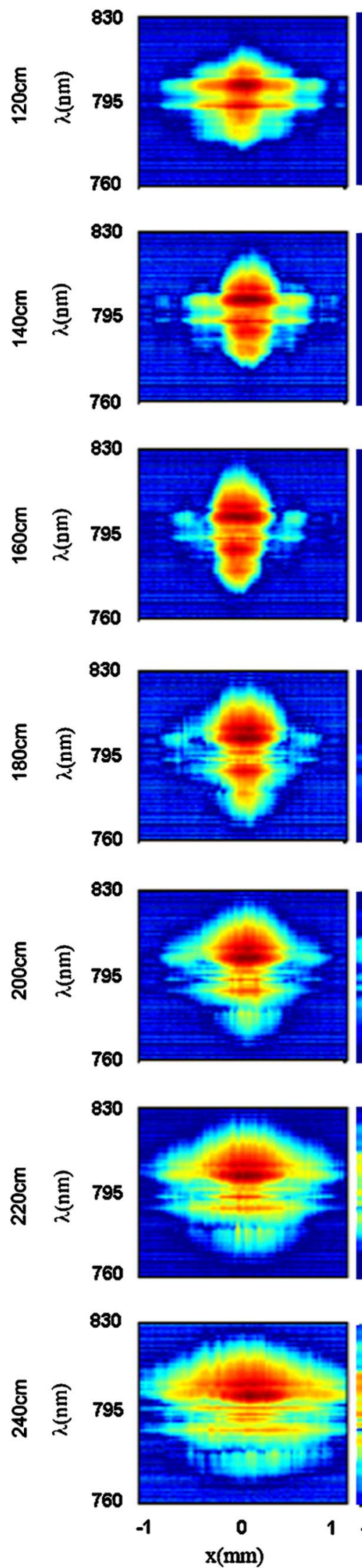

B
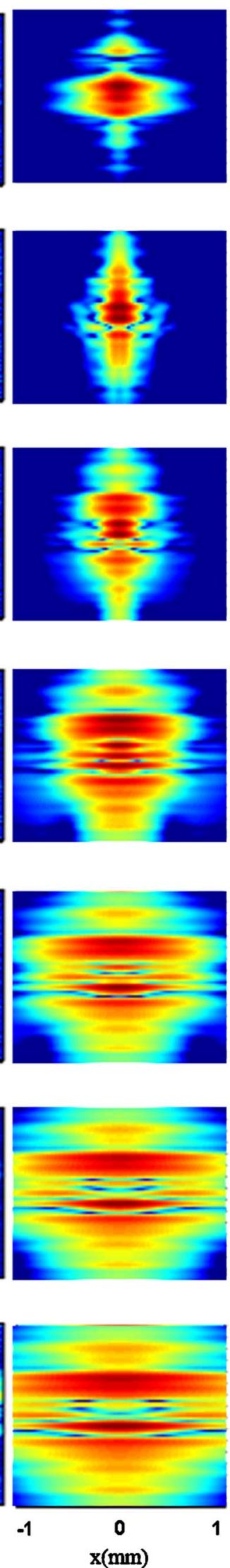

C
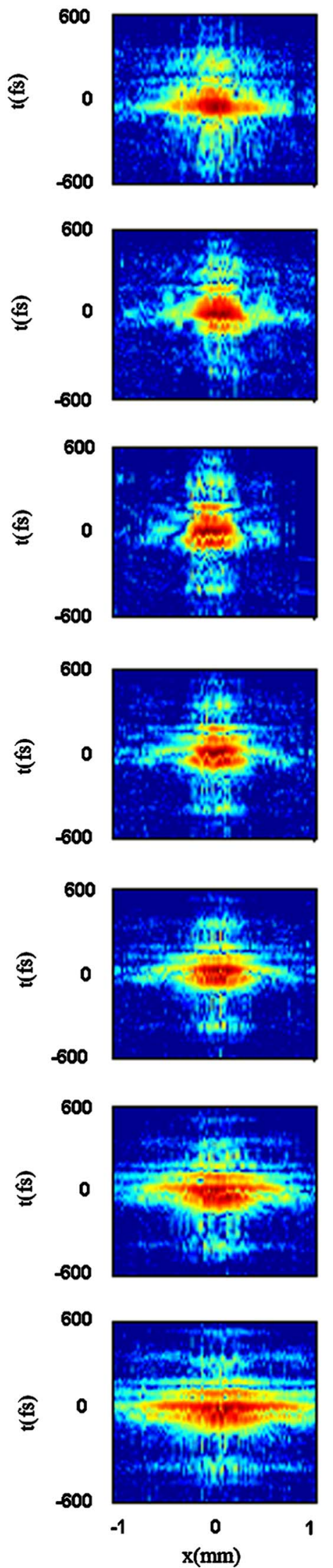

D
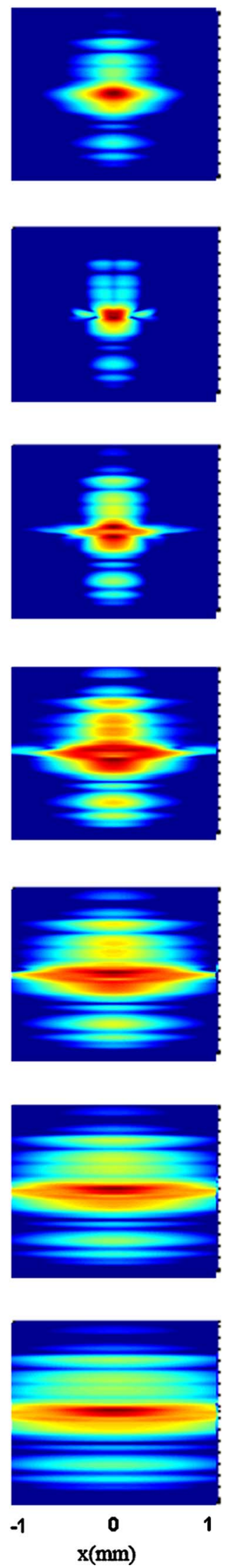

Fig. 3. (Color online) Spatially resolved spectrum, experimental (column A) and simulated (column B), and spatiotemporal intensity distribution, experimental (column C) and simulated (column D), for different propagation distances after the focusing lens, given to the left of the images. The color scale for the logarithmic plots comprises three decades. 
A
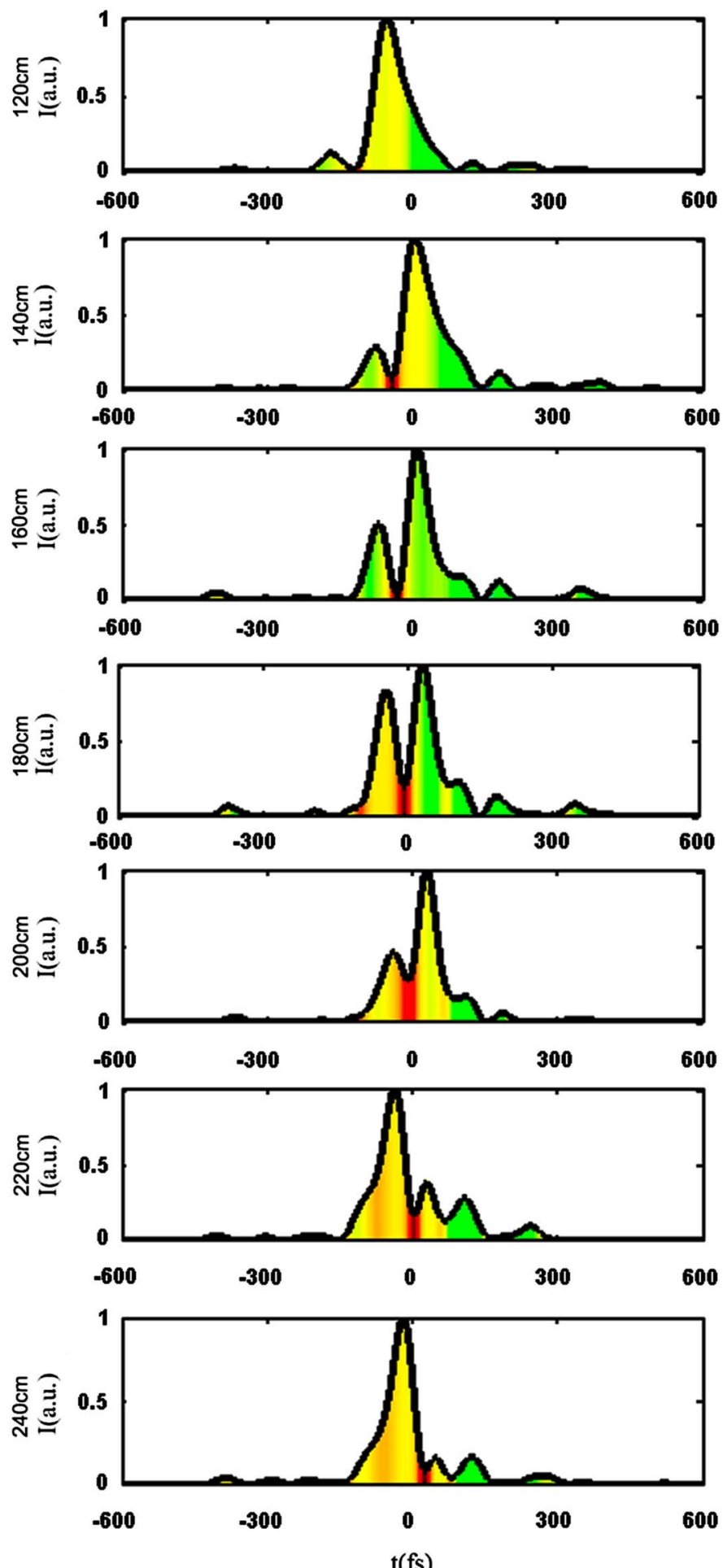

$t(f)$
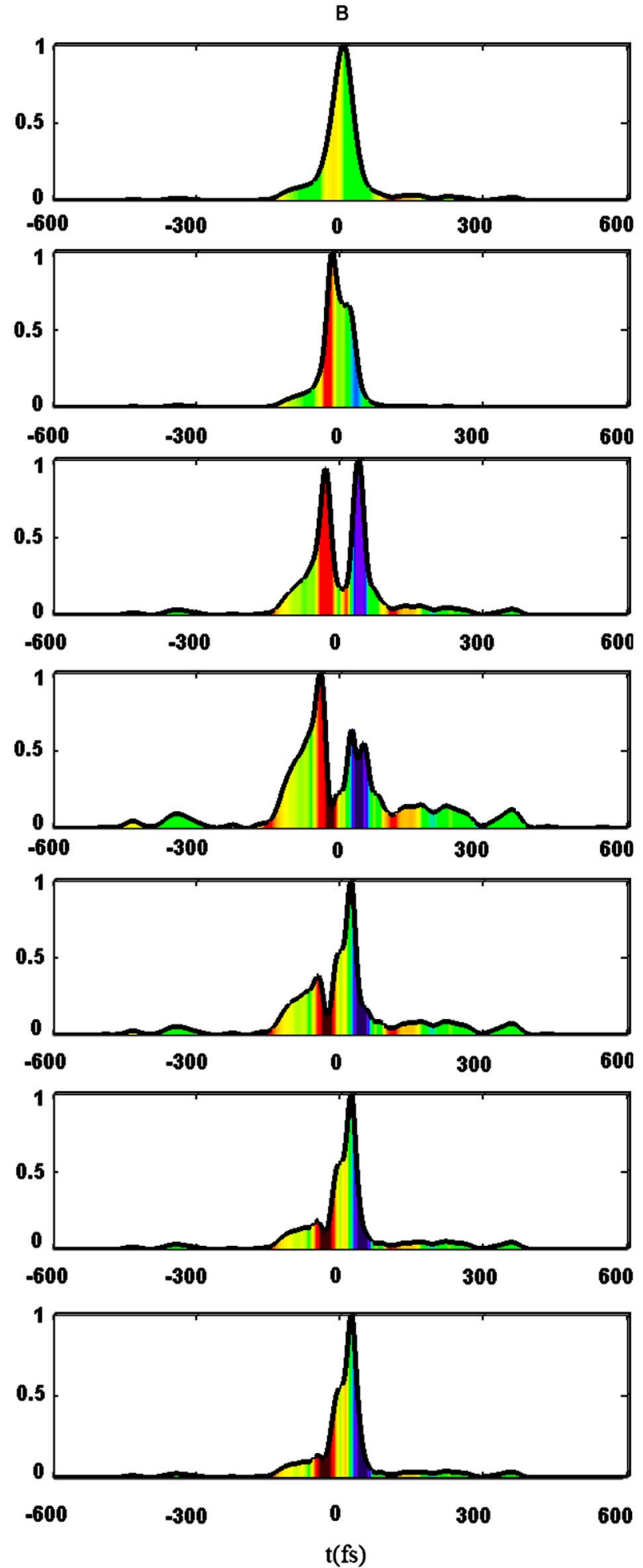

$t(f s)$

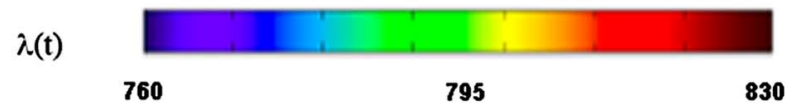

Fig. 4. (Color online) Column A, experimental, and column B, simulated on-axis pulse reconstruction for different propagation distances after the focusing lens, (indicated at the left of the figure). Color filling stands for the instantaneous wavelength.

simulation, backed by some experimental measurements, where they observed a competition between the two split pulses and that eventually the second and bluest one survived, while the first reddish one became a pedestal. In our case, we observe a similar process, despite the appearance of some differences. In our experimental case, the surviving pulse is not so blueshifted, but this may be explained due to the presence of the Raman effect on air and the relatively long pulses 


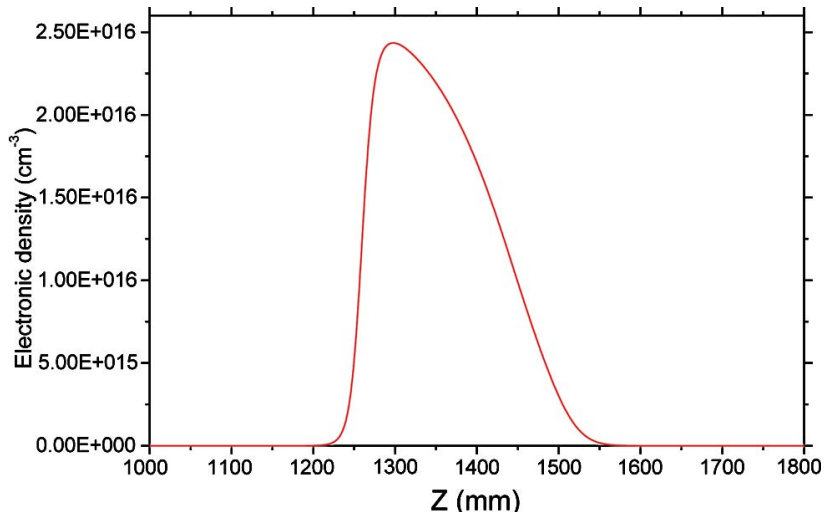

Fig. 5. (Color online) On-axis electronic density generated by the pulse along its propagation obtained in the theoretical simulations.

(longer than the typical response of the retarded Kerr effect), inducing a redshift compensation.

The temporal structure obtained for the single remaining pulse is in good agreement with the recently reported results of self-compression controlled by the input chirp [51]. After adjusting the chirp for obtaining self-compressed pulses at a given propagation position, analyzed with a SPIDER device, the temporal reconstruction of the pulses and its spectral distribution was very similar (while more compressed in the case reported in Ref. [50]) to those obtained in this work at $z=240 \mathrm{~cm}$

In general, the simulations and the experimental results present a reasonably good qualitative agreement, despite the approximations made in the model and despite the fact that it is not possible to include all the experimental parameters in the theoretical analysis (e.g., slight astigmatism, etc.). The structure and propagation evolution of the pulse front ( $r$ - $t$ reconstruction) are very similar, with the exception of the size of the beam, especially at the focalization region, where the simulation overestimates the beam collapse. From the spectral point of view, and related to the beam size difference, the simulation shows more broadened spectra than the experiments. Apart from this point, the nonlinear dynamics observed in the experiment and those obtained from the simulation are quite similar, showing the spatial collapse, the subsequent temporal pulse splitting related to the ionization of the medium, and the rich energy competition between the intensity peaks in the generated structure. In the case we have studied, the theoretical and the experimental observations show that most of the pulse energy is taken by only one temporal maximum, obtaining a single pulse output, sometimes linked with the self-compression process [50,51]. We would like to remark here that the achieved qualitative agreement confirms that the experimental procedure allows us to observe the complex spatiotemporal evolution of a laser pulse under nonlinear and self-guiding conditions. In addition, the understanding and experimental characterization of this process would be useful for the optimization of processes such as self-compression.

Concerning the quantitative discrepancies between experiments and theory, very recently Kolesik et al. [52] have shown some disagreements between experimental observations and theoretical model predictions. They did a comparison between experimental observations and two different theoretical models: the standard one (the one used in this work) and the alternative one based on the intensity-dependent Kerr ef- fect model [53]. According to their report, the experimental results presented larger filament beam dimensions (around a factor of 2) than the results calculated by both theoretical models, which induced a theoretical overestimation of the filamentation effect, showing a remarkably lower filament creation threshold than that observed in the experiments. These observations match quite well with our results: the simulations presented here show, for the same input condition as in the experiments, a smaller beam radius (around one-half of the radius measured in the experiments) and, as a consequence, a more dramatic nonlinear propagation than in the experiments. Therefore, our results, in agreement with those from Ref. [52], suggest that the theoretical model cannot achieve a quantitative agreement with the experimental results. Nevertheless, these differences do not prevent us from observing the main features of the nonlinear spatiotemporal dynamics by comparing experimental and theoretical data, obtaining an insight into the propagation dynamics within the filamentation regime, which is the aim of this work.

\section{CONCLUSION}

An experimental setup based on spatially resolved spectral interferometry using an optical fiber coupler (STARFISH) is presented, allowing the spatiotemporal reconstruction of nonlinear propagating light pulses. The nonlinear propagation of the pulse in the self-guiding regime is fully described, presenting the complex evolution of the pulse front and showing good agreement with theoretical simulations. The pulse splitting process and the subsequent dynamics of the pulses are discussed and shown, in qualitative agreement with the simulations. We believe that this kind of reconstruction opens the gate to a full understanding of the underlying physics in a broad range of nonlinear optics phenomena, obtaining intimate information about the field evolution.

\section{ACKNOWLEDGMENTS}

We acknowledge support from the Spanish Ministerio de Ciencia e Innovación through the Consolider Program Science and Applications of Ultrafast and Ultra-intense Lasers (SAUUL) (CSD2007-00013) and Research Project FIS2009-09522; from Junta de Castilla y León through the Program for Groups of Excellence (GR27) and Research Project SA002B08; and from the European Community's Seventh Framework Programme (LASERLAB-EUROPE) grant agreement 228334). We also acknowledge support from the Centro de Laseres Pulsados (CLPU), Salamanca, Spain. B. Alonso and I. J. Sola acknowledge the support of the Spanish Ministerio de Ciencia e Innovación through the "Formación de Profesorado Universitario" and "Ramón y Cajal" grant programs, respectively.

\section{REFERENCES}

1. J. San Roman, C. Ruiz, J. A. Pérez, D. Delgado, C. Mendez, L. Plaja, and L. Roso, "Nonlinear Young's double-slit experiment," Opt. Express 14, 2817-2824 (2006).

2. K. D. Moll, A. L. Gaeta, and G. Fibich, "Self-similar optical wave collapse: observation of the Townes profile," Phys. Rev. Lett. 90, 203902 (2003).

3. C. Ruiz, J. San Roman, C. Mendez, V. Diaz, L. Plaja, I. Arias, and L. Roso, "Observation of spontaneous self-channeling of light in air below the collapse threshold," Phys. Rev. Lett. 95, 053905 (2005).

4. S. L. Chin, S. A. Hosseini, W. Liu, Q. Luo, F. Théberge, N. Aközbek, A. Becker, V. P. Kandidov, O. G. Kosareva, and 
H. Schroeder, "Propagation of powerful femtosecond laser pulses in optical media: physics, applications, and new challenges," Can. J. Phys. 83, 863-905 (2005)

5. A. Braun, G. Korn, X. Liu, D. Du, J. Squier, and G. Mourou, "Selfchanneling of high-peak-power femtosecond laser pulses in air," Opt. Lett. 20, 73-75 (1995).

6. A. Couairon and A. Mysyrowicz, "Femtosecond filamentation in transparent media," Phys. Rep. 441, 47-189 (2007).

7. L. Bergé, S. Skupin, R. Nuter, J. Kasparian, and J.-P. Wolf, "Ultrashort filaments of light in weakly ionized, optically transparent media," Rep. Prog. Phys. 70, 1633-1713 (2007).

8. C. P. Hauri, W. Kornelis, F. W. Helbing, A. Heinrich, A. Couairon, A. Mysyrowicz, J. Biegert, and U. Keller, "Generation of intense, carrier-envelope phase-locked few-cycle laser pulses through filamentation,” Appl. Phys. B 79, 673-677 (2004).

9. J. Kasparian, M. Rodríguez, G. Méjean, J. Yu, E. Salmon, H. Wille, R. Bourayou, S. Frey, Y.-B. André, A. Mysyrowicz, R. Sauerbrey, J.-P. Wolf, and L. Wöste, "White-light filaments for atmospheric analysis," Science 301, 61-64 (2003).

10. J. Kasparian and J. P. Wolf, "Physics and applications of atmospheric nonlinear optics and filamentation," Opt. Express 16, 466-493 (2008).

11. K. Stelmaszczyk, P. Rohwetter, G. Méjean, J. Yu, E. Salmon, J. Kasparian, R. Ackermann, J.-P. Wolf, and L. Wöste, "Longdistance remote laser-induced breakdown spectroscopy using filamentation in air," Appl. Phys. Lett. 85, 3977-3979 (2004).

12. S. Tzortzakis, G. Méchain, G. Patalano, Y.-B. André, B. Prade, M. Franco, A. Mysyrowicz, J.-M. Munier, M. Gheudin, G. Beaudin, and P. Encrenaz, "Coherent subterahertz radiation from femtosecond infrared filaments in air," Opt. Lett. 27, 1944-1946 (2002)

13. K. Ishikawa, H. Kumagai, and K. Midorikawa, "High-power regime of femtosecond-laser pulse propagation in silica: multiple cone formation," Phys. Rev. E 66, 056608 (2002).

14. A. Couairon, M. Franco, G. Méchain, T. Olivier, B. Prade, and A. Mysyrowicz, "Femtosecond filamentation in air at low pressures: Part I: theory and numerical simulations," Opt. Commun. 259, 265-273 (2006).

15. S. Champeaux, L. Bergé, D. Gordon, A. Ting, J. Peñano, and P. Sprangle, " $(3+1)$-dimensional numerical simulations of femtosecond laser filaments in air: toward a quantitative agreement with experiments," Phys. Rev. E 77, 036406 (2008).

16. H. Schillinger and R. Sauerbrey, "Electrical conductivity of long plasma channels in air generated by self-guided femtosecond laser pulses," Appl. Phys. B 68, 753-756 (1999).

17. J. Bernhardt, W. Liu, F. Théberge, H. L. Xu, J. F. Daigle, M. Châteauneuf, J. Dubois, and S. L. Chin, "Spectroscopic analysis of femtosecond laser plasma filament in air," Opt. Commun. 281, 1268-1274 (2008).

18. V. Loriot, E. Hertz, O. Faucher, and B. Lavorel, "Measurement of high order Kerr refractive index major air components," Opt. Express 17, 13429-13434 (2009).

19. A. Zaïr, A. Guandalini, F. Schapper, M. Holler, J. Biegert, L. Gallmann, A. Couairon, M. Franco, A. Mysyrowicz, and U. Keller, "Spatiotemporal characterization of few-cycle pulses obtained by filamentation," Opt. Express 15, 5394-5404 (2007).

20. S. Minardi, A. Gopal, A. Couairon, G. Tamoašuskas, R. Piskarskas, A. Dubietis, and P. Di Trapani, "Accurate retrieval of pulse-splitting dynamics of a femtosecond filament in water by time-resolved shadowgraphy,” Opt. Lett. 34, 3020-3022 (2009).

21. I. Blonskyi, V. Kadan, O. Shpotyuk, and I. Dmituk, "Manifestations of sub- and superluminality in filamented femtosecond laser pulse in fused silica," Opt. Commun. 282, 1913-1917 (2009).

22. J. Trull, O. Jedrkiewicz, P. Di Trapani, A. Matijošius, A. Varanavičius, G. Valiulis, R. Danielius, E. Kucinskas, A. Piskarskas, and S. Trillo, "Spatiotemporal three-dimensional mapping of nonlinear $X$ waves," Phys. Rev. E 69, 026607 (2004).

23. A. Matijošius, J. Trull, P. Di Trapani, A. Dubietis, R. Piskarskas, A. Varanavičius, and A. Piskarskas, "Nonlinear space-time dynamics of ultrashort wave packets in water," Opt. Lett. 29, 1123-1125 (2004).

24. S. Minardi, M. A. C. Potenza, and J. Trull, "Intensity mapping of three-dimensional optical wave packet: holographic properties and applications," in Trends in Laser and Electro-optical Research, W. T. Arkin, ed. (2006), pp. 151-182.

25. C. Dorrer, E. M. Kosik, and I. A. Walmsley, "Spatiotemporal characterization of the electric field of ultrashort optical pulses using two-dimensional shearing interferometry," Appl. Phys. B 74, S209-S217 (2002)

26. C. Dorrer, E. M. Kosik, and I. A. Walmsley, "Direct space-time characterization of the electric fields of ultrashort optical pulses," Opt. Lett. 27, 548-550 (2002).

27. P. Gabolde and R. Trebino, "Single-shot measurement of the full spatiotemporal field of ultrashort pulses with multispectral digital holography," Opt. Express 14, 11460-11467 (2006)

28. P. Bowlan, P. Gabolde, and R. Trebino, "Directly measuring the spatiotemporal electric field of focusing ultrashort pulses," Opt. Express 15, 10219-10230 (2007).

29. P. Bowlan, H. Valtna-Lukner, M. Lõhmus, P. Piksarv, P. Saari, and R. Trebino, "Measuring the spatiotemporal field of ultrashort Bessel- $X$ pulses," Opt. Lett. 34, 2276-2278 (2009).

30. B. Alonso, I. J. Sola, O. Varela, J. Hernández-Toro, C. Méndez, J. San Román, A. Zaïr, and L. Roso, "Spatiotemporal amplitudeand-phase reconstruction by Fourier-transform of interference spectra of high-complex beams," J. Opt. Soc. Am. B 27, 933-940 (2010).

31. O. Mendoza-Yero, B. Alonso, O. Varela, G. Mínguez-Vega, I. J. Sola, J. Lancis, V. Climent, and L. Roso, "Spatiotemporal characterization of ultrashort pulses diffracted by circularly symmetric hard-edge apertures: theory and experiment," Opt. Express 18, 20900-20911 (2010)

32. C. G. Durfee, D. E. Adams, and J. A. Squier, "Spatiotemporal characterization of ionizing wavefronts in a filament," presented at Third International Symposium on Filamentation, Crete, Greece, 31 May-5 June 2010.

33. J. Piasecki, B. Colombeau, M. Vampouille, C. Froehly, and J. A. Arnaud, "Nouvelle méthode de mesure de la réponse impulsionnelle des fibers optiques," Appl. Opt. 19, 3749-3755 (1980).

34. L. Lepetit, G. Cheriaux, and M. Joffre, "Linear techniques of phase measurement by femtosecond spectral interferometry for applications in spectroscopy," J. Opt. Soc. Am. B 12, 2467-2474 (1995).

35. C. Iaconis and I. A. Walmsley, "Spectral phase interferometry for direct electric-field reconstruction of ultrashort optical pulses," Opt. Lett. 23, 792-794 (1998).

36. R. Trebino, K. DeLong, D. Fittinghoff, J. Sweester, M. Krumbügel, B. Richman, and D. Kane, "Measuring ultrashort laser pulses in the time-frequency domain using frequency-resolved optical gating," Rev. Sci. Instrum. 68, 3277-3295 (1997).

37. P. O'Shea, M. Kimmel, X. Gu, and R. Trebino, "Highly simplified device for ultrashort-pulse measurement," Opt. Lett. 26, 932-934 (2001)

38. T. Brabec and F. Krausz, "Nonlinear optical pulse propagation in the single-cycle regime," Phys. Rev. Lett. 78, 3282-3285 (1997).

39. A. Couairon, S. Tzortzakis, L. Berge, M. Franco, B. Prade, and A. Mysyrowicz, "Infrared femtosecond light filaments in air: simulations and experiments," J. Opt. Soc. Am. B 19, 1117-1131 (2002).

40. M. Mlejnek, E. M. Wright, and J. V. Moloney, "Dynamic spatial replenishment of femtosecond pulses propagating in air," Opt. Lett. 23, 382-384 (1998)

41. L. Berge and A. Couairon, "Gas-induced solitons," Phys. Rev. Lett. 86, 1003-1006 (2001).

42. S. Skupin, L. Bergé, U. Peschel, and F. Lederer, "Interaction of femtosecond light filaments with obscurants in aerosols," Phys. Rev. Lett. 93, 023901 (2004).

43. A. Couairon and A. Mysyrowicz, "Femtosecond filamentation in transparent media," Phys. Rep. 441, 47-189 (2007).

44. L. Bergé, S. Skupin, F. Lederer, G. Méjean, J. Yu, J. Kasparian, E. Salmon, J. P. Wolf, M. Rodriguez, L. Wöste, R. Bourayou, and R. Sauerbrey, "Multiple filamentation of terawatt laser pulses in air," Phys. Rev. Lett. 92, 225002 (2004).

45. L. Bergé, S. Skupin, and G. Steinmeyer, "Self-recompression of laser filaments exiting a gas cell," Phys. Rev. A 79, 033838 (2009). 
46. J. Zhang, Z. H. Lu, and L. J. Wang, "Precision refractive index measurements of air, $\mathrm{N}_{2}, \mathrm{O}_{2}, \mathrm{Ar}$, and $\mathrm{CO}_{2}$ with a frequency comb," Appl. Opt. 47, 3143-3151 (2008).

47. C. R. Giuliano, J. H. Marburger, and A. Yariv, Appl. Phys. Lett. 21, 58-60 (1972).

48. M. Mlejnek, E. M. Wright, and J. V. Moloney, "Moving focus versus self-waveguiding model for long distance propagation of femtosecond pulses in air," IEEE J. Quantum Electron. 35 1771-1776 (1999).

49. C. Bree, A. Demircan, S. Skupin, L. Bergé, and G. Steinmeyer, "Plasma-induced pulse breaking in filamentary selfcompression," Laser Phys. 20, 1107 (2010).

50. S. Skupin, G. Stibenz, L. Bergé, F. Lederer, T. Sokollik, M. Schnürer, N. Zhanvoronkov, and G. Steinmeyer, "Self- compression by femtosecond pulse filamentation: experiments versus numerical simulations," Phys. Rev. E 74, 056604 (2006).

51. O. Varela, B. Alonso, I. J. Sola, J. San Román, A. Zaïr, C. Méndez, and L. Roso, "Self-Compression controlled by the chirp of the input pulse," Opt. Lett. 35, 3649-3652 (2010).

52. M. Kolesik, D. Mirell, J.-C. Diels, and J. V. Moloney, "On the higher-order Kerr effect in femtosecond filaments," Opt. Lett. 35, 3685-3687 (2010).

53. P. Bejot, J. Kasparian, S. Henin, V. Loriot, T. Vieillard, E. Hertz, O. Faucher, B. Lavorel, and J.-P. Wolf, "Higher-Order Kerr terms allow ionization-free filamentation in gases," Phys. Rev. Lett. 104, 103903 (2010). 